\title{
Special issue on Botryosphaeriaceae
}

\author{
AJL Phillips \\ University of Lisbon, Faculty of Sciences, Biosystems and Integrative Sciences Institute (BioISI), Campo Grande, 1749- \\ 016 Lisbon, Portugal \\ E-mail: alan.jl.phillips@gmail.com
}

AJL Phillips 2016 - Special issue on Botryosphaeriaceae. Mycosphere 7(7), 868-869, Doi 10.5943/mycosphere/si/1b/12

Since its introduction in 1918, the family Botryosphaeriaceae has attracted considerable attention from mycologists and plant pathologists, it has also been the source of much confusion. For many years its taxonomic position was undecided and only in 2007 was it finally established in its own order, Botryosphaeriales. The number of species in the family increased dramatically in the early years of the 1900s, mostly because species were introduced on the basis of host-association. The number of genera also increased dramatically during the years when separate names were used for different stages of the life-cycle of a single fungus. It was only around the early 2000s, when DNA sequence-based phylogenies became routine, that some order was restored to the family. These techniques proved invaluable for resolving cryptic species complexes and for clarifying the status of genera and species. They have now become essential for species identification, especially in the larger genera, such as Diplodia, Lasiodiplodia and Neofusicoccum.

Despite the invaluable contribution they have made towards the resolution of taxonomic problems, DNA sequence-based phylogenetic analyses have also introduced several complications. At present there is no consensus on which loci should be used to define species, or how many differences constitute a distinct species. Within Botryosphaeriaceae it is clear that ITS and tefl- $\alpha$ sequences can resolve almost all species even in such large genera as Neofusicoccum. Nevertheless, with the tendency towards giving species names for individual lineages in phylograms, there is the danger that some species names may in fact represent genetic variations within a species. As a result of the increased interest in Botryosphaeriaceae, together with the ease of introducing new genus and species names, the family is presently undergoing another period of expansion. Over the last three years, six genera and 85 new species have been introduced.

The present issue of Mycosphere comprises two essays and ten papers that represent a cross-section of the current interest in this family. In the first essay Chetana et al. (p. 870) emphasize the importance of using the correct name for genera and species, because names are the primary means for communicating information. The second essay by Manawasinghe et al. (p. 883) highlights how little we know about the pathology of Botryosphaeriaceae or what triggers the change from an endophytic to a parasitic lifestyle. It also points out that the methods currently used for pathogenicity testing are inadequate. Linaldeddu et al. (p. 893) studied the species associated with a serious disease of urban trees and introduce a new genus to accommodate the causal agent. Lopes et al. (p. 906) revealed the diversity of Neofusicoccum species in forest and urban environments. Surveys of relatively under-sampled regions continue to reveal new species in Botryosphaeriaceae. This is exemplified by the new species introduced in the genera Barriopsis (Konta et al. p. 921), Phaeobotryon (Darangama et al. p. 933), Spencermartinsia (Zhang et al. p. 
942), Neodeightonia (Konta et al. p. 950) and Diplodia (Linaldeddu et al. p. 962). As shown by Giambra et al. (p. 978), new species continue to be found in relatively well-studied regions and hosts. Tennakoon et al. (p. 990) report on and provide an illustrated description of the sexual morph of Lasiodiplodia pseudotheobromae. Related to this is the report that Barriopsis archontophoenicis can form the sexual morph in culture (Konta et al. p. 921). In the final paper Dissanayake et al. (p. 1001) provide an updated list of genera and species in Botryosphaeriaceae together with details of types, geographical distribution and known hosts. 